\title{
Confidence-based Contractor, Propagation and Potential Clouds for Differential Equations*
}

\author{
Julien Alexandre dit Sandretto ${ }^{a}$
}

\begin{abstract}
A novel interval contractor based on the confidence assigned to a random variable is proposed in this paper. It makes it possible to consider at the same time an interval in which the quantity is guaranteed to be, and a confidence level to reduce the pessimism induced by interval approach. This contractor consists in computing a confidence region. Using different confidence levels, a particular case of potential cloud can be computed. As application, we propose to compute the reachable set of an ordinary differential equation under the form of a set of confidence regions, with respect to confidence levels on initial value.
\end{abstract}

Keywords: interval analysis, confidence level, potential cloud, reachability for ODEs

\section{Introduction}

An interval (see [16] for more details) aims to bound all the values of an uncertain quantity, for example provided by a measurement device [11]. This approach is highly effective for every safety, verification or validation procedures because intervals are conservative. The major inconvenience is that intervals are sometimes too pessimistic, and lead to unexploitable results. Obviously, bounds can be set on a given measured datum (arbitrarily large), therefore the measurement can be guaranteed to be enclosed in an interval. However, a probability distribution can also be deduced from past observations, with more effort, and associated to the measurement device. We propose to exploit a probability distribution to reduce the pessimism of intervals.

In order to filter (or reduce) an interval with respect to a given information (such as a constraint, a measurement or any kind of information), contractors are mainly used [5]. A contractor is a function taking an interval as input and

\footnotetext{
*This work was supported by the "Chair Complex Systems Engineering - Ecole polytechnique, THALES, DGA, FX, Dassault Aviation, DCNS Research, ENSTA Paris, Télécom Paris, and Fondation ParisTech" and partially supported by DGA AID.

${ }^{a}$ ENSTA Paris, Institut Polytechnique de Paris, Palaiseau, France. E-mail: alexandre@ensta.fr, ORCID: https://orcid.org/0000-0002-6185-2480.
} 
returning a smaller interval (included in the previous one). It can be seen as a filtering approach in the sense that a contractor reduces an interval without any solution loss. Contractors are often associated to a propagation procedure, at least a propagation loop, to communicate a contraction on a variable to the others through some constraints.

In this paper, a novel contractor is proposed to filter an interval following a confidence level given on the associated quantity. This confidence level is an input of the contractor, the "new" information, while the probability distribution of the considered variable is a characteristic of the associated random variable. To compute such contraction, the probability density function (or density for short) is taken into account. In this paper, we then focus on random continuous variable with a known (and analytical) density function, such as uniform distribution, normal distribution, beta distribution, etc.

Combining intervals and probability has been already proposed in numerous papers using techniques such as p-boxes [8, 21], fuzzy sets [7, 18], box-particles [1] and potential cloud $[9,18]$. Some of these representations can be deduced from probability intervals [6]. The notion of cloud is interesting for us to represent a very substantial result to a problem such as computing the image of a function, a set inversion, or the solution of a constraint satisfaction problem (these problems have been solved with interval methods in [11]). Computing a solution of such a problem with several confidence levels provides different boxes which, gathered, provide a particular type of potential cloud.

We are particularly interested in Ordinary Differential Equations (ODEs) and validated methods to compute their reachable sets via validated simulation $[3,12,17,20]$. In the case of Initial Value Problems (IVPs) with ODEs, the initial state is primordial. An uncertain initial state is generally bounded in a box (also in a zonotope [3] or a polytope [4]). As experimentation, we propose to consider in addition to this initial box some confidence levels, and we apply the presented approach. It allows us to describe the reachable set by a cloud. This more expressive result can then be used in various control problems, parameter identification, verification, etc.

This paper is organized as follows. The next section is dedicated to establishing the notation and recall the notion of confidence in probability theory. Section 3 presents the relationship we use between probabilities and interval analysis. Sections 4 and 5 contain the main results presented in this article: a confidence-based contractor and the propagation of a confidence contraction to a potential cloud. The last section concludes the article and gives some hint on future works.

\section{Confidence Interval}

In this section, some notions required for the definition of confidence interval are introduced. However, to clarify the concept of confidence interval as soon as possible, an informal explanation can be given: 
A confidence interval is a set $\mathcal{S}$ for which the probability of the given random variable that lies in this set is equal to the given probability $P$.

Let us define $X$ a random variable (also called random quantity, aleatory variable). A random variable takes different values, resulting from a random phenomenon. In this paper, we focus on continuous univariate distributions such that $X \in \mathbb{R}$. Such a variable implies a mapping (a function) between its possible values and a probability of appearance. It means that $X$ is measurable.

Definition 1 (Continuous random real variable). A continuous random variable is a random variable whose cumulative distribution function is continuous everywhere [14].

Considering a continuous random real variable $X: \Omega \mapsto \mathcal{A}, X$ is a measurable function from a set of possible outcomes $\Omega$ to the measurable space $\mathcal{A}$.

The probability that $X$ takes a value in a measurable subset $S \subset \mathcal{A}$ is given by:

$$
\operatorname{Pr}[X \in S]=P(\{\omega \in \Omega \mid X(\omega) \in S\}),
$$

where $P$ is the probability measure equipped with $\Omega$.

Considering continuous variable, $X$ can take any numerical value in an interval following the distribution. The distribution is then fully characterized by a probability density function (see Figure 1).

Definition 2 (Probability density function). A random variable $X$ with values in a measurable space (usually $\mathbb{R}^{n}$ ) has probability density function $f_{X}$, where $f_{X}$ is a non-negative Lebesgue-integrable function, if:

$$
\operatorname{Pr}[a \leq X \leq b]=\int_{a}^{b} f_{X}(x) d x .
$$

A property is fundamental in probability:

$$
\operatorname{Pr}[-\infty<X<\infty]=\int_{-\infty}^{\infty} f_{X}(x) d x=\mathbf{1}
$$

Let us define $\hat{x}$ a single observed sample of the quantity $X$. In statistics, an observed datum allows to compute a confidence interval [19], that is to say an interval which may contain the actual value, with respect to a given confidence level. A formal definition can then be stated:

Definition 3 (Confidence interval). Let $X$ be a random sample from a probability distribution $f_{X}$. A confidence interval with confidence level cc is an interval with endpoints $a$ and $b$ with the property:

$$
\operatorname{Pr}(a<X<b)=\int_{a}^{b} f_{X}(x) d x=c c .
$$




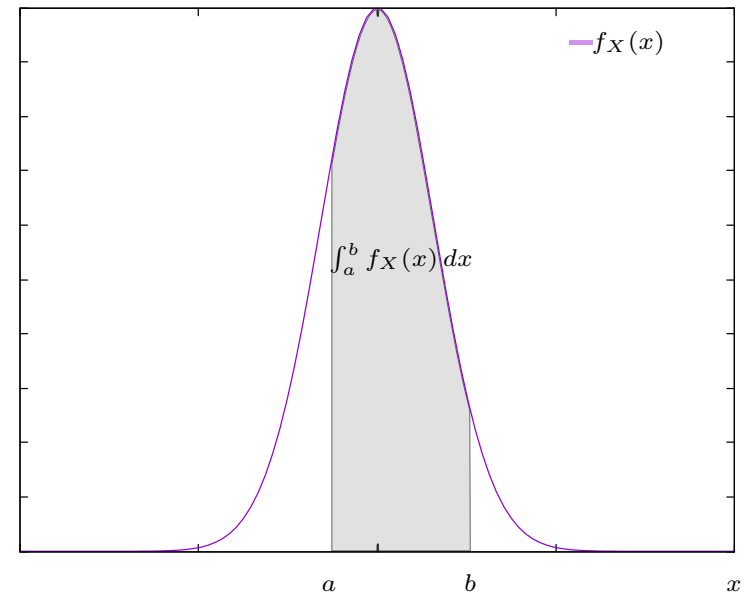

Figure 1: Probability density function of a variable $X$.

For example, considering a confidence level $C L=95 \%$, one can define the confidence interval $C_{95 \%}$. This interval can be obtained by observation (statistical approach) or with the help of a known distribution (probability approach). A new measurement $\hat{x}$ coming from the (same) experiment will be in the associated confidence interval such that:

$$
\hat{x} \in C_{95 \%} \quad 95 \% \text { of the time. }
$$

Figure 2 illustrates the concept of confidence interval and confidence level for a normal distribution.

In the particular case of symmetric distribution and regarding centered confidence intervals, they follow the inclusion property:

$$
C L_{1}<C L_{2} \Longrightarrow C_{C L_{1}} \subset C_{C L_{2}}
$$

For example, $C_{90 \%} \subset C_{95 \%}$.

Remark 1. The extremal values for a confidence level have a particular meaning: $0 \%$ means that there is no chance that a future observation will follow the previous observations, while $100 \%$ means that it is sure that a future observation will follow the previous observations.

In the following, the distribution are considered symmetric. Therefore, we mainly focus on normal dstribution. 


\section{Interval Analysis and Probability}

\subsection{Introduction to intervals}

The simplest and most common way to represent and manipulate sets of values is interval arithmetic (see $[15])$. An interval $\left[x_{i}\right]=\left[x_{i}, \overline{x_{i}}\right]$ defines the set of reals $x_{i}$ such that $x_{i} \leq x_{i} \leq \overline{x_{i}}$. $\mathbb{I R}$ denotes the set of all intervals over reals. The size or the width of $\left[x_{i}\right]$ is denoted by $w\left(\left[x_{i}\right]\right)=\overline{x_{i}}-x_{i}$.

Interval arithmetic extends to $\mathbb{I} \mathbb{R}$ elementary functions over $\mathbb{R}$. For instance, the interval sum, i.e., $\left[x_{1}\right]+\left[x_{2}\right]=\left[x_{1}+x_{2}, \overline{x_{1}}+\overline{x_{2}}\right]$, encloses the image of the sum function over its arguments. An interval vector or a box $[\mathbf{x}] \in \mathbb{I} \mathbb{R}^{n}$, is a Cartesian product of $n$ intervals. The enclosing property basically defines what is called an interval extension or an inclusion function.

Definition 4 (Inclusion function). Consider a function $f: \mathbb{R}^{n} \rightarrow \mathbb{R}^{m}$, then $[f]$ : $\mathbb{I} \mathbb{R}^{n} \rightarrow \mathbb{R}^{m}$ is said to be an extension of $f$ to intervals if

$$
\forall[\mathbf{x}] \in \mathbb{I}^{n}, \quad[f]([\mathbf{x}]) \supseteq\{f(\mathbf{x}), \mathbf{x} \in[\mathbf{x}]\} .
$$

It is possible to define inclusion functions for all elementary functions such as $\times, \div, \sin , \cos$, exp, etc. The natural inclusion function is the simplest to obtain: all occurrences of the real variables are replaced by their interval counterpart and all arithmetic operations are evaluated using interval arithmetic. More sophisticated inclusion functions such as the centered form, or the Taylor inclusion function may also be used (see [11] for more details).

Combining the inclusion function and the rectangle rule, integral can be bounded following:

$$
\int_{a}^{b} f(x) d x \in(b-a) \cdot[f]([a, b])
$$

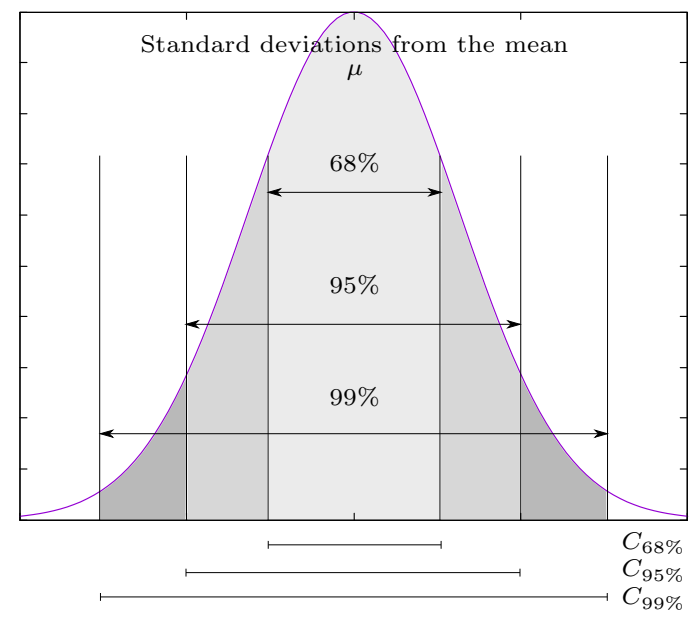

Figure 2: Confidence intervals and confidence levels. 


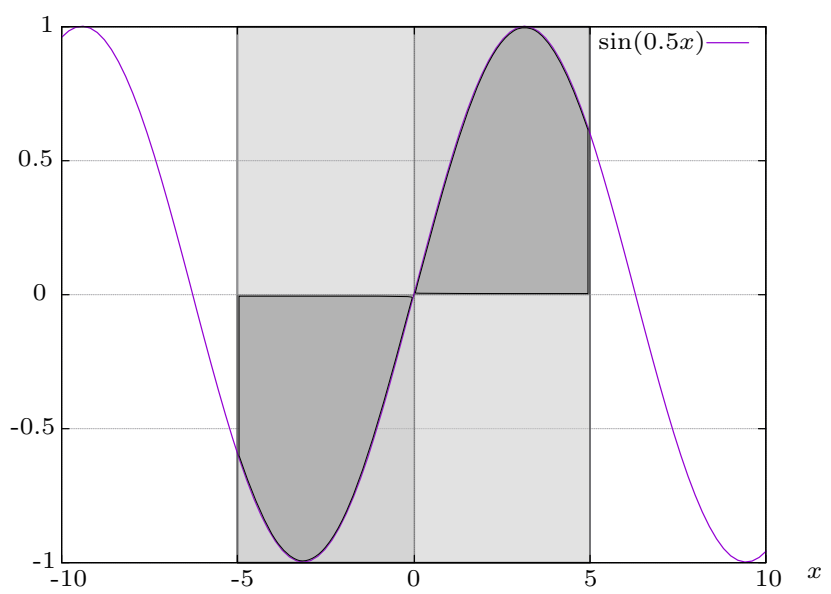

Figure 3: Computation of $\int_{-5}^{5} \sin (0.5 x) d x$. With interval $[-5,5]$ (in light grey), result is $[-10,10]$ while with two intervals $([-5,0],[0,5])$ (in darker grey) we obtain $[-5,5]$.

In order to obtain a better approximation, a discretization of the integral can be used, as shown in Figure 3:

$$
\int_{a}^{b} f(x) d x=\sum_{i=1}^{n} \int_{k_{i}}^{k_{i+1}} f(x) d x \in \sum_{i=1}^{n}\left(k_{i+1}-k_{i}\right) \cdot[f]\left(\left[k_{i}, k_{i+1}\right]\right),
$$

with $k_{1}=a$ and $k_{n+1}=b$.

Notations: In the following, we denote by $1.2[3,4]$ the interval $[1.23,1.24]$.

\subsection{Intervals and probability}

A random variable $X$ with a probability density $f_{X}$ is observed via a measurement device. We consider that the density is defined by $\mu$, a mean or expectation of the distribution, and $\sigma$ a standard deviation. An observed sample is denoted by $\hat{x}$, given with the device associated uncertainty $\pm m$. An interval containing the actual value can be defined as $[x]=[\hat{x}-m, \hat{x}+m]$ (bias can also be added). For example, we consider that $X$ follows a normal distribution, such that

$$
f_{X}(x \mid \mu, \sigma)=\frac{1}{\sqrt{2 \pi \sigma^{2}}} e^{-\frac{(x-\mu)^{2}}{2 \sigma^{2}}}
$$

with $\mu=1.0$ and $\sigma=1.0$ (then the variance $\sigma^{2}=1.0$ ). Interval arithmetic and Equation (2) allow us to compute an enclosure of the integral of the density between 0 and 1 (i.e., the probability $\operatorname{Pr}[0 \leq X \leq 1]$ ), as depicted in Figure 4. The computed result with $n=100$ is $0.34[05578,21275]$ (while a mathematical tool ${ }^{1}$ using floating

\footnotetext{
${ }^{1}$ Matlab was used for this comparison.
} 
numbers computes 0.341345$)$. Using symmetry, the integral between 0 and 2 , that is to say between $\mu-\sigma$ and $\mu+\sigma$, is included in $0.68[11,42]$, which contains the theoretical confidence level $(68.27 \%)$ for the confidence interval $[\mu-\sigma, \mu+\sigma]$.

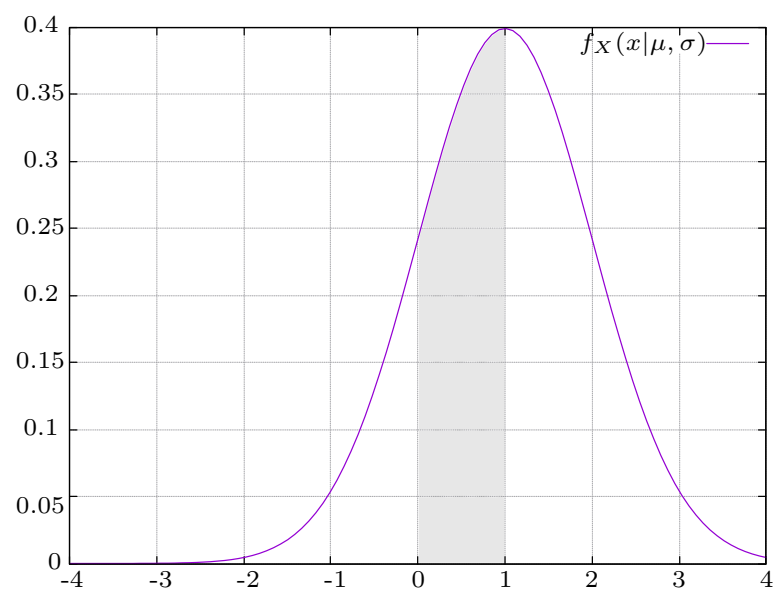

Figure 4: Interval computation of the integral of a probability density (for a normal distribution).

In theory, about $95 \%$ of the values lie within two standard deviations, that is to say in the interval $[\mu-2 \sigma, \mu+2 \sigma]$. With interval analysis, the obtained probability is $0.9[48,61]$, with $n=100$, while with $n=1000$ it is reduced to $0.95[38,51]$ (the approximation is better).

Remark 2. The extremal values for a confidence level have a particular meaning (see Remark 1). We define the associated confidence intervals such that $C_{0 \%}=\emptyset$ and $C_{100 \%}=[-\infty, \infty]$.

\section{Confidence-based Contractor}

The main idea of our contribution is that a measurement provides an interval (by considering the uncertainty of the measurement device) which is guaranteed to contain the actual quantity ${ }^{2}$, but sometimes too pessimistic to be workable. In addition to an enclosure, the observed variable can be associated to a probability distribution. Our idea is to combine an interval provided by a measurement and the probability distribution by establishing a confidence level on the quantity.

\subsection{A confidence-based contractor}

A generic contractor $\mathrm{Cr}$ must satisfy two properties [11]:

- Contractance : $\forall[x] \in \mathbb{R}, C r([x]) \subset[x]$,

\footnotetext{
${ }^{2}$ Outliers are not considered in this paper.
} 
- Correctness : $\forall[x] \in \mathbb{R},[x] \cap \mathbb{S} \subseteq C r([x])$ (with $\mathbb{S}$ the solution set).

We propose a confidence-based contractor, denoted $C b c$, defined as follows:

$$
\begin{aligned}
C b c\left([x] \mid f_{X}, c c\right): \quad & \mathbb{I R} \mapsto \mathbb{R} \\
& {[x] \rightarrow[x] \cap[y] }
\end{aligned}
$$

with $[y]$ defined such that $\operatorname{Pr}(x \in[y])=\int_{[y]} f_{X}(x) d x=c c([y]$ is the confidence interval), $c c$ being the confidence coefficient $(0 \leq c c \leq 1)$. For example, one can use the parameter assignment $c c=0.68$ for a confidence level of $68 \%$.

Proposition 1. The confidence-based contractor is a contractor.

Proof. Two properties have to hold: contractance and correctness. The contractance is obvious because the confidence-based contractor uses the intersection operation, and $[x] \cap[y] \subseteq[x], \forall[y]$. Correctness is more complex to handle as a new type of correctness needs to be introduced: the confidence correctness. Confidence correctness means that if the confidence given on the quantity is well estimated for a sample, then the variable lies in the corresponding confidence interval with the associated probability and then the correctness holds. Therefore, if the correctness may not hold, the confidence correctness holds (all computations are conducted with validated interval arithmetic). For example, the 90\%-correctness holds, except for $10 \%$ of the samples.

Figure 5 illustrates the effect of the confidence-based contractor applied to the following example:

Example 1. Let $X$ a random variable with a normal distribution, such that

$$
f_{X}(x \mid \mu, \sigma)=\frac{1}{\sqrt{2 \pi \sigma^{2}}} e^{-\frac{(x-\mu)^{2}}{2 \sigma^{2}}}
$$

with $\mu=1.0$ and $\sigma=1.0$. The quantity $X$ is observed and one measurement is obtained: $[x]=[0.7,2.1]$. A confidence level of $68.27 \%$ is given on $X$, that is to say that we are confident on the accuracy of the observations, so $X$ stays close to its mean. Our method computes the contraction such that:

$$
\begin{aligned}
C b c([0.7,2.1] \mid(1.0,1.0), 0.6827) & =[0.7,2.1] \cap[0.0,2.0] \\
& =[0.7,2.0]
\end{aligned}
$$

So the upper bound is reduced with respect to the confidence level. The pessimism induced by interval approach is thus limited.

As seen before, two special cases can be described:

- $\forall[x], C b c\left([x] \mid f_{X}, 0\right)=\emptyset$ (annihilating element)

- $\forall[x], C b c\left([x] \mid f_{X}, 1\right)=[x]$ (identity element) 


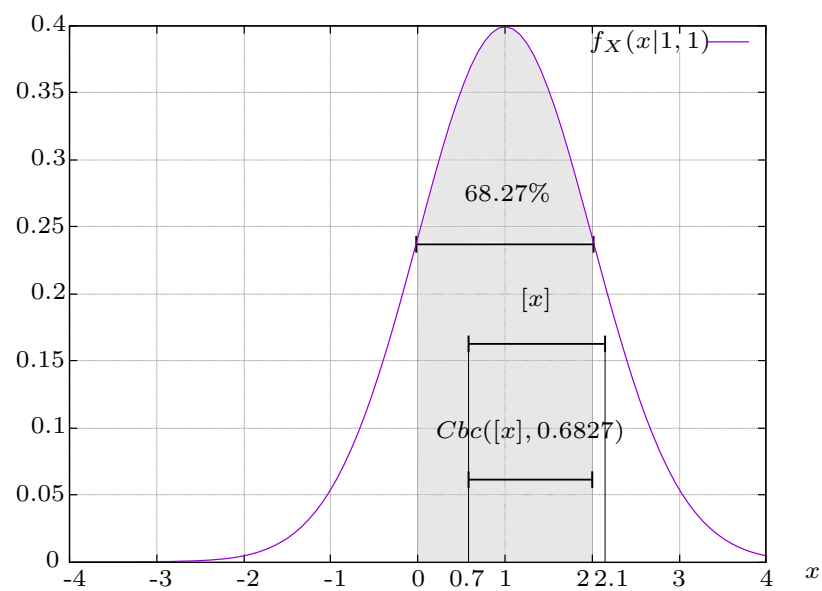

Figure 5: Illustration of confidence-based contraction.

For two different confidence coefficients $c c_{1}$ and $c c_{2}$ such that $c c_{1}<c c_{2}$, the following order holds:

$$
\forall[x], C b c\left([x] \mid f_{X}, c c_{1}\right) \subset C b c\left([x] \mid f_{X}, c c_{2}\right)
$$

The contractor $C b c$ can be composed with other contractors or with itself. The order previously shown leads to two particularities:

- $\forall[x], C b c\left(C b c\left([x] \mid f_{X}, c c_{2}\right) \mid f_{X}, c c_{1}\right)=C b c\left([x] \mid f_{X}, c c_{1}\right)$

- $\forall[x], C b c\left(C b c\left([x] \mid f_{X}, c c_{1}\right) \mid f_{X}, c c_{2}\right)=C b c\left([x] \mid f_{X}, c c_{1}\right)$

That is to say that the lower confidence coefficient is primary. The standard operations on sets can be extended to this specific contractor:

- $\left(C b c\left([x] \mid f_{X}, c c_{1}\right) \cap C b c\left([x] \mid f_{X}, c c_{2}\right)\right)([x])=C b c\left([x] \mid f_{X}, c c_{1}\right)$ (intersection)

- $\left(C b c\left([x] \mid f_{X}, c c_{1}\right) \cup C b c\left([x] \mid f_{X}, c c_{2}\right)\right)([x])=C b c\left([x] \mid f_{X}, c c_{2}\right)$ (union)

Remark 3. As a confidence interval is enclosed by its support interval (which guarantee the enclosure of the quantity), outliers can be automatically detected and rejected. Our approach can then be able to produce robust confidence intervals.

\subsection{Computation of confidence interval}

The confidence-based contractor presented in this paper needs the computation of the confidence interval associated to a given confidence level. Three cases can be detailed:

- Case 1: a well known probability distribution and a particular confidence level with known confidence interval. For example, a normal distribution with a $95 \%$ confidence level gives a confidence interval $[\mu-2 \sigma, \mu+2 \sigma]$. 
- Case 2: a probability distribution with a known inverse function, such as the inverse of error function for Gaussian density function (i.e. $\operatorname{erf}^{-1}$ ).

- Case 3: the general symmetric case without any particular values.

We focus on the third case with the presentation of Algorithm 1. This algorithm follows a predictor-corrector based approach. This latter needs guesses $a$ and $b$. For mean-centered confidence intervals, $a$ and $b$ have to be chosen such that $\mu=\frac{a+b}{2}$ (but it is not mandatory, in presence of bias for example). Two operations are also needed to implement this algorithm: Narrow and Widen. Widen is equivalent to an inflation of a well chosen percentage (e.g. 1\%), while Narrow is a deflation of the same percentage.

We apply the proposed algorithm to Example 1 for different confidence levels. The results are gathered in Table 1 and plotted in Figure 6. Experiments are as follows: for different confidence levels $c c_{i}$ from $10 \%$ to $99 \%$ - and with the particular value $68.27 \%$ - (first column of Table 1), we apply the contractor $C b c\left([-5,5] \mid f_{X}, c c_{i}\right)$, with $f_{X}$ from Example 1. It provides several contracted confidence intervals (second column of Table 1). Then, for all these confidence intervals, we compute the probability for the variable to be in the confidence interval with the method presented in Section 3.2 (third column of Table 1) for verification.

Remark 4. In the case of a non symmetric distribution, the maximal confidence interval can be computed to obtain a one-to-one application between a confidence coefficient $c c$ and confidence interval $[a, b]$. The maximal confidence interval is defined by:

$$
\max _{[a, b]}\|b-a\|, \quad \operatorname{Pr}[a \leq X \leq b]=c c
$$

\section{Application to Reachability}

As an application, we propose to compute the reachability of Ordinary Differential Equations (ODEs) from an interval initial value. We imagine that the initial value

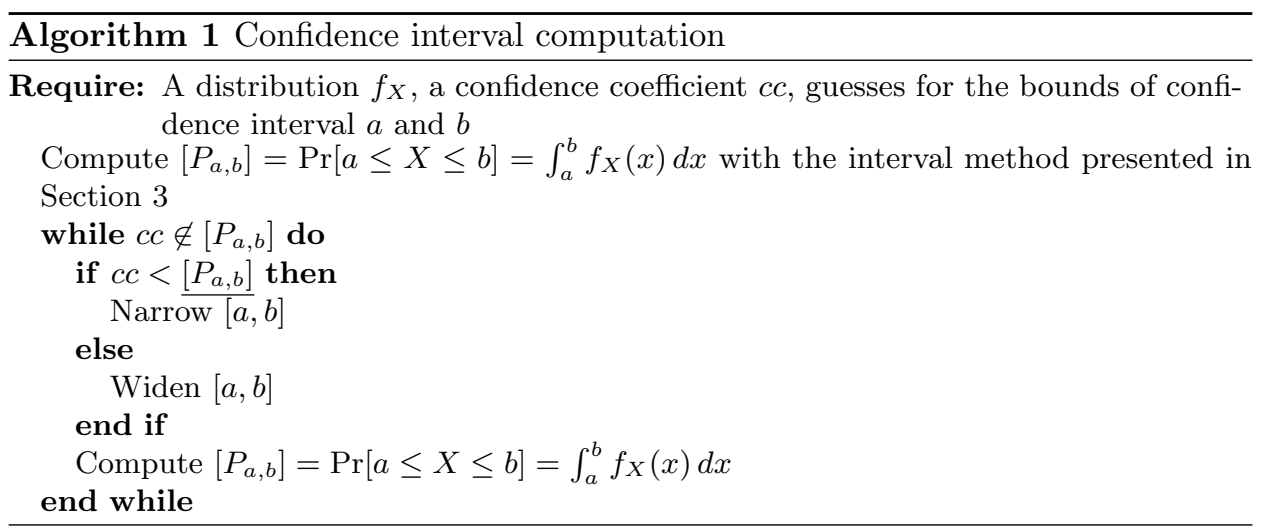


Table 1: Confidence intervals for different values of confidence level and computed probabilities.

\begin{tabular}{ccc} 
Confidence & Interval & Probability \\
\hline $10 \%$ & {$[0.8743,1.1256]$} & {$[0.0999,0.1000]$} \\
$20 \%$ & {$[0.7466,1.2533]$} & {$[0.1999,0.2000]$} \\
$30 \%$ & {$[0.6146,1.3853]$} & {$[0.2999,0.3000]$} \\
$40 \%$ & {$[0.4756,1.5243]$} & {$[0.3999,0.4000]$} \\
$50 \%$ & {$[0.3256,1.6743]$} & {$[0.4998,0.5000]$} \\
$60 \%$ & {$[0.1581,1.8418]$} & {$[0.5999,0.6003]$} \\
$68.27 \%$ & {$[0,2]$} & {$[0.6824,0.6830]$} \\
$70 \%$ & {$[-0.0362,2.0362]$} & {$[0.6995,0.7002]$} \\
$80 \%$ & {$[-0.2800,2.2800]$} & {$[0.7988,0.8000]$} \\
$90 \%$ & {$[-0.6450,2.6450]$} & {$[0.8990,0.9010]$} \\
$95 \%$ & {$[-0.9500,2.9500]$} & {$[0.9475,0.9501]$} \\
$99 \%$ & {$[-1.5200,3.5200]$} & {$[0.9863,0.9901]$}
\end{tabular}

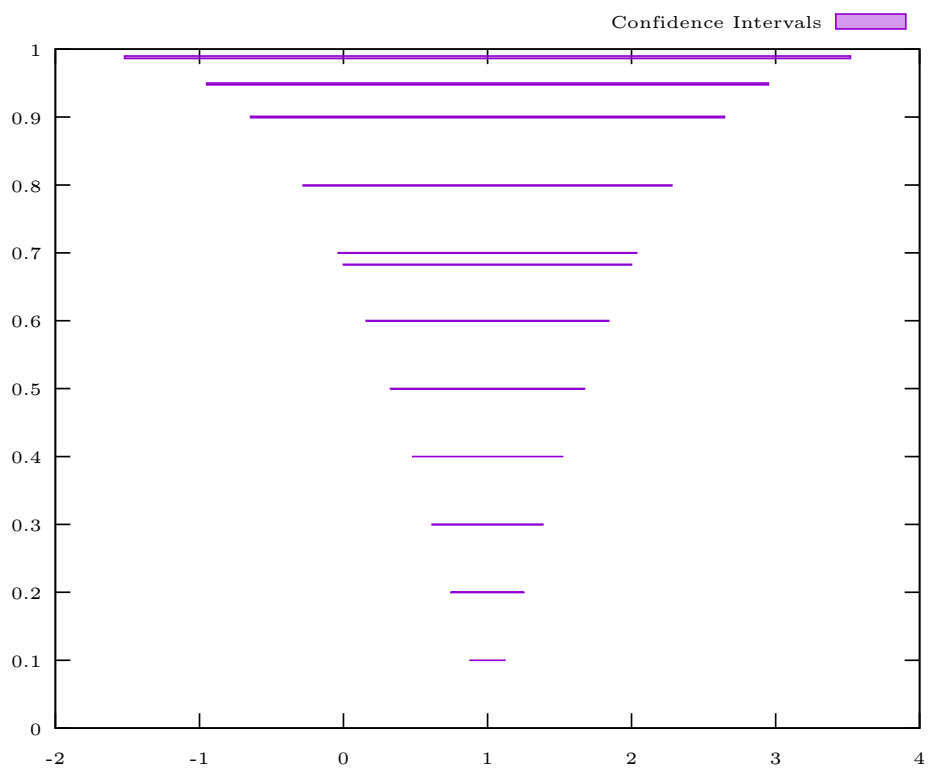

Figure 6: Confidence intervals for different values of confidence level with respect to computed probabilities.

is provided by a measurement and that different confidence levels can be considered. The proposed confidence-based contractor can then be used to reduce the initial interval. We solve the Initial Value Problem (IVP) for all the obtained intervals by the help of validated integration. The collection of computed reachable sets is 
depicted through a kind of potential cloud that is useful for verification, control synthesis, or validation problems on ODEs. An approach consisting also in the propagation of probabilities through an initial value problem with ODE has been proposed in last decade [13]. If the goal is the same, how to add information coming from a probability knowledge to the reachability analysis, the technique is different. In [13], authors exploit Taylor models to reprensent uncertainties (mainly on parameters), and propagate them into an integration process to compute fuzzy trajectories (see [22] for fuzzy sets). Our contribution is mainly based on confidence level, this concept is not considered in [13]. Nevertheless, an example from this latter is studied in Section 5.3 to compare and discuss both approaches.

\subsection{Integration and propagation}

\subsubsection{Validated simulation}

When dealing with validated computation, mathematical representation of an IVPODE is as follows:

$$
\left\{\begin{array}{l}
\dot{\mathbf{y}}(t)=g(t, \mathbf{y}(t)) \\
\mathbf{y}(0) \in\left[\mathbf{y}_{0}\right] \subseteq \mathbb{R}^{n}
\end{array}\right.
$$

We assume that $g: \mathbb{R} \times \mathbb{R}^{n} \rightarrow \mathbb{R}^{n}$ is continuous in $t$ and globally Lipschitz in $\mathbf{y}$, so Equation (4) admits a unique solution.

The set (expressed as a box) $\left[\mathbf{y}_{0}\right]$ of initial conditions is used to model some (bounded) uncertainties. For a given initial condition $\mathbf{y}_{0} \in\left[\mathbf{y}_{0}\right]$, the solution at time $t>0$, when it exists, is denoted $\mathbf{y}\left(t ; \mathbf{y}_{0}\right)$. The goal, for validated numerical integration methods, is then to compute the set of solutions of Equation (4), i.e., the set of possible solutions at time $t$ given the initial condition in the set of initial conditions $\left[\mathbf{y}_{0}\right]$ :

$$
\mathbf{y}\left(t ;\left[\mathbf{y}_{0}\right]\right)=\left\{\mathbf{y}\left(t ; \mathbf{y}_{0}\right) \mid \mathbf{y}_{0} \in\left[\mathbf{y}_{0}\right]\right\} .
$$

Validated numerical integration schemes, exploiting set-membership framework, aim at producing the solution of the IVP-ODE that is the set defined in Equation (5). It results in the computation of an outer approximation of $\mathbf{y}\left(t ;\left[\mathbf{y}_{0}\right]\right)$. The use of set-membership computation for the problem described above makes possible the design of an inclusion function for the computation of $[\mathbf{y}]\left(t ;\left[\mathbf{y}_{0}\right]\right)$, which is an outer approximation of $\left.\mathbf{y}\left(t ; \mathbf{y}_{0}\right]\right)$ defined in Equation (5). To do so, a sequence of time instants $t_{1}, \ldots, t_{n}$ such that $t_{1}<\cdots<t_{n}$ and a sequences of boxes $\left[\mathbf{y}_{1}\right], \ldots,\left[\mathbf{y}_{n}\right]$ such that $\mathbf{y}\left(t_{i+1} ;\left[\mathbf{y}_{i}\right]\right) \subseteq\left[\mathbf{y}_{i+1}\right], \forall i \in[0, n-1]$ are computed. From $\left[\mathbf{y}_{i}\right]$, computing the box $\left[\mathbf{y}_{i+1}\right]$ is a classical 2-step method (see [12]):

- Phase 1: compute an a priori enclosure $\left[\tilde{\mathbf{y}}_{i}\right]$ of the set $\left\{\mathbf{y}\left(t_{k} ; \mathbf{y}_{i}\right) \mid t_{k} \in\right.$ $\left.\left[t_{i}, t_{i+1}\right], \mathbf{y}_{i} \in\left[\mathbf{y}_{i}\right]\right\}$, such that $\mathbf{y}\left(t_{k} ;\left[\mathbf{y}_{i}\right]\right)$ is guaranteed to exist,

- Phase 2: compute a tight enclosure of the solution $\left[\mathbf{y}_{i+1}\right]$ at time $t_{i+1}$.

Two main approaches can be used to compute the tight enclosure in Phase 2. The first one, and the most used, is the Taylor method [15, 17]. The second one, more 
recently studied, is the validated Runge-Kutta approach [3]. The reachability consists in computing the enclosure of the set of states at a specific instant $\tau$ as defined above by $\left[\mathbf{y}(\tau) ;\left[\mathbf{y}_{0}\right]\right]$.

\subsubsection{Propagation}

The procedure given in Section 5.1.1, applied to Equation (5), produces a reachable tube based on time discretization as depicted in Figure 7. Considering an initial value $\left[\mathbf{y}_{0}\right]^{*}$ such that $\left[\mathbf{y}_{0}\right]^{*} \subseteq\left[\mathbf{y}_{0}\right]$, a propagation procedure $[2]$ can be performed to compute the solution of the IVP-ODE:

$$
\left\{\begin{array}{l}
\dot{\mathbf{y}}(t)=g(t, \mathbf{y}(t)) \\
\mathbf{y}(0) \in\left[\mathbf{y}_{0}\right]^{*} \subseteq \mathbb{R}^{n} .
\end{array}\right.
$$

by keeping in mind the fact that $\mathbf{y}\left(t ;\left[\mathbf{y}_{0}\right]^{*}\right) \subseteq \mathbf{y}\left(t ;\left[\mathbf{y}_{0}\right]\right), \forall t$. We propagate along the discretization the contraction of the initial state with the help of a Runge-Kutta based contractor as proposed in [3]. The resulting reachable tube is showed in Figure 7.

One simulation followed by several propagation is much faster than several simulations [2] due to the economy of the dynamic discretization re-computation and the conservation of the first phase results (which is the more time-consuming step).

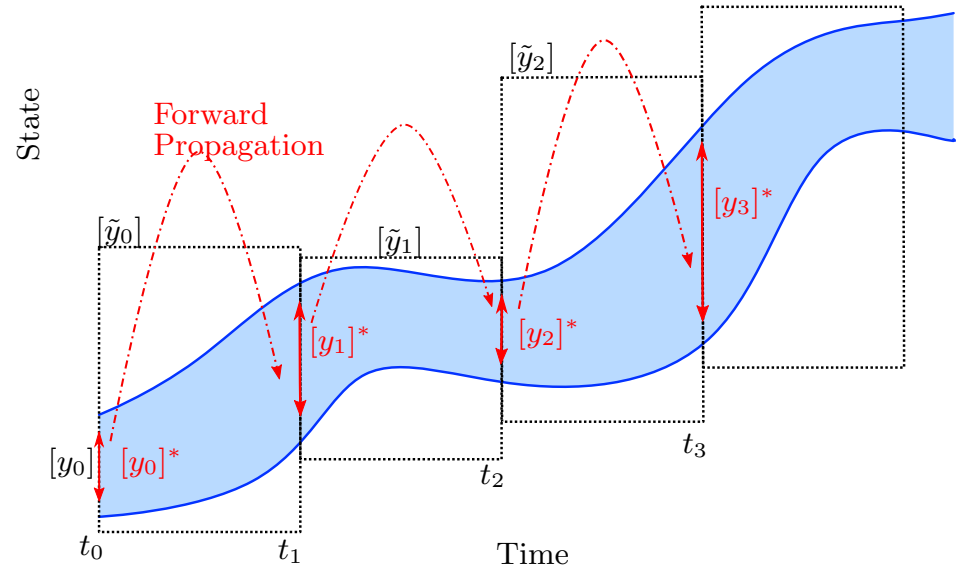

Figure 7: Continuous reachable tube (in blue) and propagation (in red) of a new initial condition $\left[y_{0}\right]^{*}$ (with $\left.\left[y_{0}\right]^{*} \subset\left[y_{0}\right]\right)$.

\subsection{Potential clouds}

The formalism of clouds has been proposed in [18] to handle uncertainties. With clouds, uncertainties are seen as safety constraints. The potential clouds can be exploited for high dimensional and non-formalised uncertainties, as in [10]. 
From [18], the formal definition of a cloud over a set $\mathcal{M}$ is a mapping $\mathbf{x}$ that associates with each $\xi \in \mathcal{M}$ a nonempty, closed and bounded interval $\mathbf{x}(\xi)$ such that

$$
] 0,1\left[\subseteq \bigcup_{\xi \in \mathcal{M}} \mathbf{x}(\xi) \subseteq[0,1] .\right.
$$

$\mathbf{x}(\xi)=[\underline{x}(\xi), \bar{x}(\xi)]$ is called the level of $\xi$ in the cloud $\mathbf{x}$. A cloud and an $\alpha$-cut are illustrated in Figure 8.

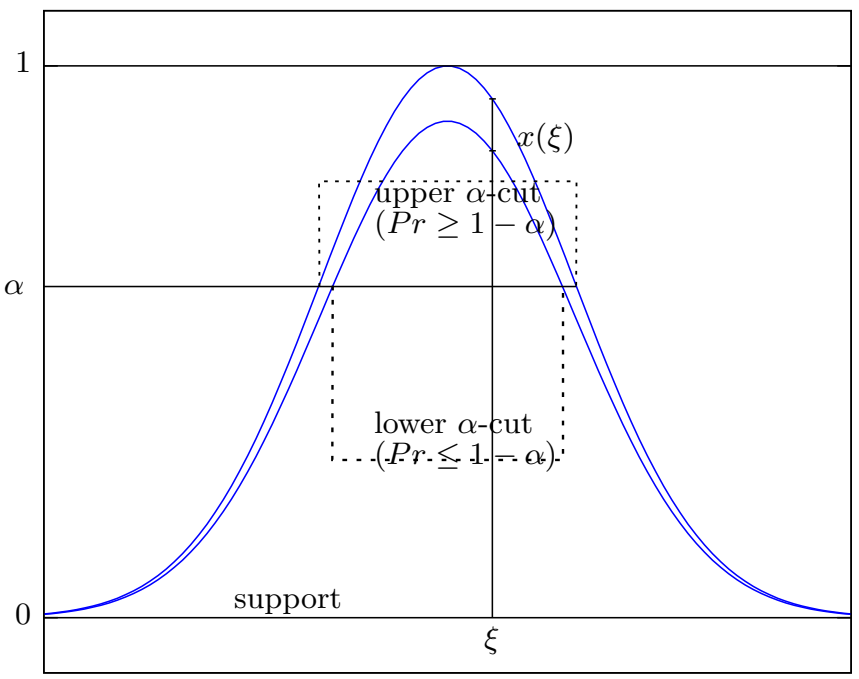

Figure 8: A cloud over $\mathbb{R}$ with an $\alpha$-cut at $\alpha=0.6$.

In the particular case where a cloud $\mathbf{x}$ is defined by a potential function $V$ : $\mathcal{M} \mapsto \mathbb{R}$ (bounded below) such that

$$
\mathbf{x}(\xi):=[\operatorname{Pr}(V(x)>V(\xi)), \operatorname{Pr}(V(x) \geq V(\xi))]
$$

that can be written $\mathbf{x}(\xi):=[\underline{\alpha}(V(\xi)), \bar{\alpha}(V(\xi))]$ where $\alpha$-cuts are level sets of $V$. In general, the probabilities are not known and then $\underline{\alpha}, \bar{\alpha}: \mathbb{R} \mapsto[0,1]$ are assumed (we call these functions potential level maps), and $\mathbf{x}$ is called a potential cloud.

Regarding the definition, determining a cloud is similar to compute a lower and an upper bounds of the confidence regions for different confidence levels (a discretization from $100 \%$ to $10 \%$ for example) with the help of Cumulative Distribution Functions (CDFs). Considering multivariate problems, a potential function is used to map a multivariate random variable to a univariate one. In the following, we consider only the upper bound of the confidence regions because our main interest concerns safety.

Proposition 2. The collection of the reachable sets $\left[\mathbf{y}\left(\tau ;\left[\mathbf{y}_{0}\right]^{i}\right)\right], i=1 \ldots m$, with $\left[\mathbf{y}_{0}\right]^{i}=C b c\left(\left[\mathbf{y}_{0}\right] \mid f_{X}, c c_{i}\right)$ is a special case of potential clouds applied to reachability. 
In order to illustrate this capability, a one-dimensional example is studied (multivariate problems can be considered by the help of a potential function).

Example 2. The following IVP is considered:

$$
\left\{\begin{array}{l}
\dot{y}(t)=y \cos (y) \\
y(0) \in[0,2]
\end{array}\right.
$$

The initial condition follows a normal distribution, such that

$$
f_{X}(x \mid \mu, \sigma)=\frac{1}{\sqrt{2 \pi \sigma^{2}}} e^{-\frac{(x-\mu)^{2}}{2 \sigma^{2}}}
$$

with $\mu=1.0$ and $\sigma=1.0$ (same as before, recalled for clarity). The system described by Equation (9) has to reach a goal given by the interval $[1.5,1.6]$ at $t=5$, i.e., $\left[y\left(5 ;\left[y_{0}\right]\right)\right] \subset[1.5,1.6]$. Our objective is to prove that the system reaches its goal with respect to a confidence level given on the initial condition. Equation (9) is solved with validated simulation as described in [3]. The obtained reachable set at $t=5$ is $[-276.986,279.276]$. The goal is then unfulfilled. With successive tests from a confidence of $90 \%$ to $10 \%$ (from pessimistic to optimistic), the confidence-based contractor is applied followed by a forward propagation along the validated simulation (as presented in Section 5.1.2).

The reachable sets of problems described by:

$$
\left\{\begin{array}{l}
\dot{y}(t)=y \cos (y) \\
y(0) \in\left[y_{0}\right]^{i}=C b c\left([0,2] \mid f_{X}, c c_{i}\right)
\end{array}\right.
$$

are gathered in Table 2 .

Table 2: Confidence levels, contracted initial intervals and reachable sets.

\begin{tabular}{ccc} 
Confidence & Initial & Final \\
\hline $90 \%$ & {$[0,2]$} & {$[-276.986,279.276]$} \\
$80 \%$ & {$[0,2]$} & {$[-276.986,279.276]$} \\
$70 \%$ & {$[0,2]$} & {$[-276.986,279.276]$} \\
$60 \%$ & {$[0.1581,1.8418]$} & {$[-189.871,192.408]$} \\
$50 \%$ & {$[0.3256,1.6743]$} & {$[1.56281,1.57764]$} \\
$40 \%$ & {$[0.4756,1.5243]$} & {$[1.56871,1.57205]$} \\
$30 \%$ & {$[0.6146,1.3853]$} & {$[1.56964,1.57119]$} \\
$20 \%$ & {$[0.7466,1.2533]$} & {$[1.57004,1.57082]$} \\
$10 \%$ & {$[0.8743,1.1256]$} & {$[1.57027,1.57061]$}
\end{tabular}

It shows that:

- Goal can be proved to be achieved after $50 \%$, i.e., we have one chance out of two that the initial state is in the contracted interval; 
- Confidence contractor has effect from $60 \%$;

- Contraction-propagation approach reduces computation time (106 seconds for ten simulations versus 87 seconds for one simulation and nine propagations).

The trajectories for $50 \%$ and $60 \%$ are given in Figure 9. A potential cloud composed by the reachable sets for different confidence levels is depicted in Figure 10.

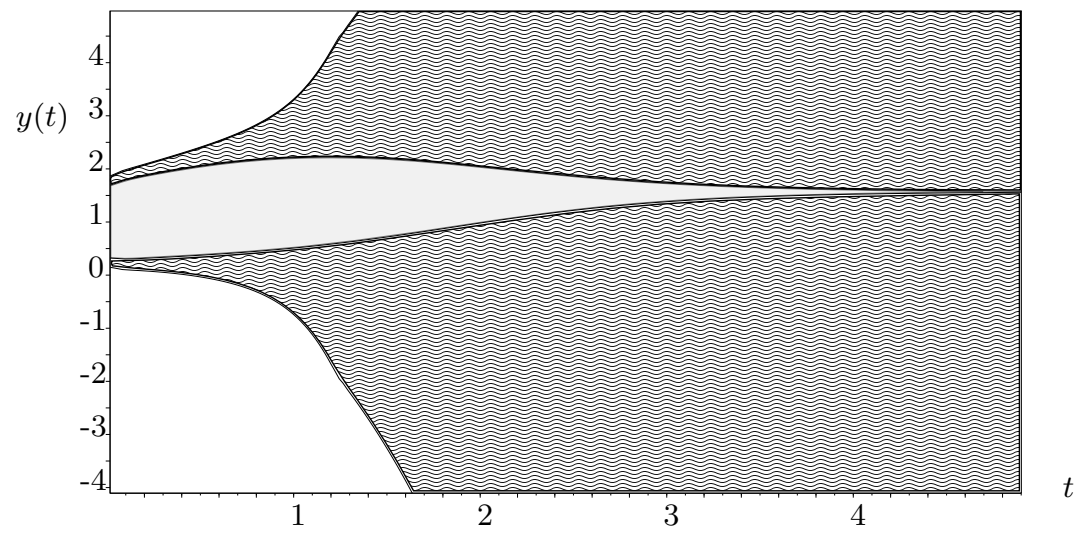

Figure 9: Validated trajectories for $60 \%$ (in grey waves) and for $50 \%$ (in light grey) confidence contraction.

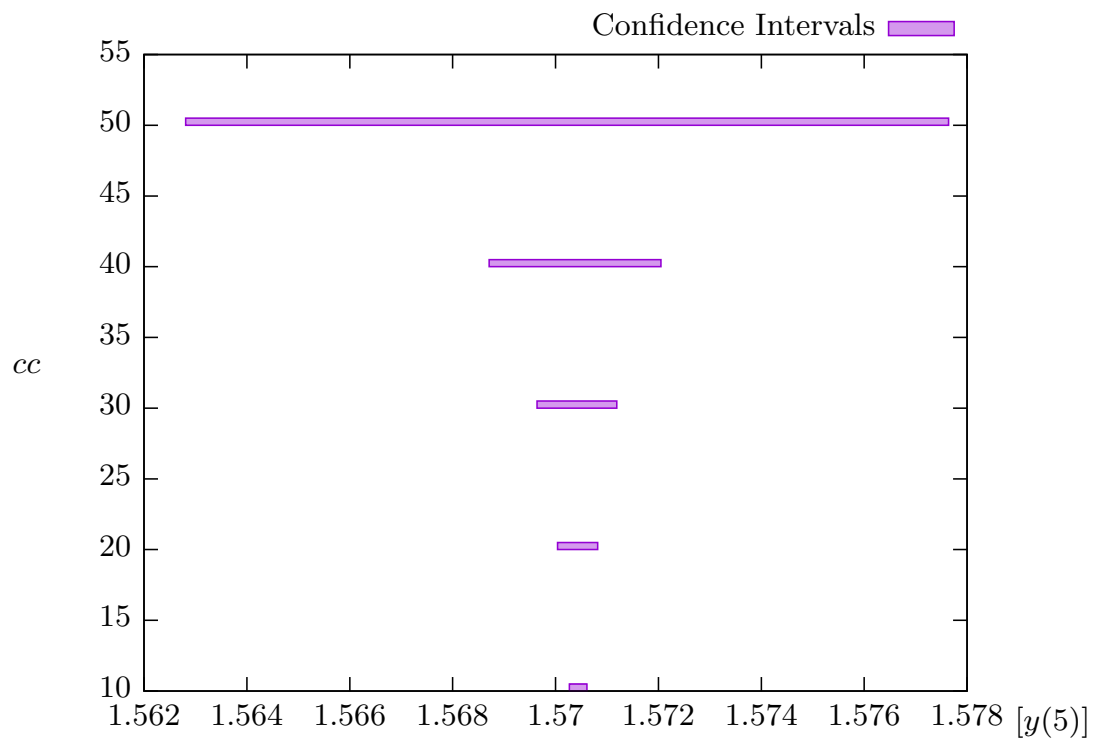

Figure 10: Reachable set for different confidence levels presented as a potential cloud. 


\subsection{Comparison with trapezoidal fuzzy numbers}

In [13], authors propose several interesting examples. In particular, a two-state bioreactor model with uncertain parameters is studied. It consists in a well-mixed bioreactor in which biomass of a single organism is produced with respect to a single limiting substrate. The continuous dynamics is described by:

$$
\left\{\begin{array}{l}
\dot{X}=(\mu-a D) X \\
\dot{S}=D\left(S_{f}-S\right)-k \mu X,
\end{array}\right.
$$

where $X$ and $S$ are the concentrations of biomass and substrate. Here $\mu$ is a function of $S$ describing the specific growth rate of biomass given by $\mu=\frac{\mu_{\max } S}{K_{s}+S}$ (in the case of monod kinetics), $D$ is the dilution rate, $a$ the biomass washout fraction, $k$ the inverse yield coefficient, and $S_{f}$ the substrate feed concentration. The parameter values are: $a=0.5, k=10.53, S_{f}=5.7 \mathrm{~g} / \mathrm{L}$ and $\mu_{\max }=1.2 \mathrm{~h}^{-1}$. The initial states are: $X(0)=0.829 \mathrm{~g} / \mathrm{L}$ and $S(0)=0.8 \mathrm{~g} / \mathrm{L}$. The two parameters $D$ and $K_{s}$ are treated as uncertain and represented by symmetric trapezoidal fuzzy numbers in [13]. This kind of fuzzy number is described by its support and core intervals. For $D$, support and core are $[0.35,0.37] \mathrm{h}^{-1}$ and $[0.35667,0.36333] \mathrm{h}^{-1}$ (respectively), and for $K_{s}$ they are $[6.8,7.2] \mathrm{g} / \mathrm{L}$ and $[6.93333,7.06667] \mathrm{g} / \mathrm{L}$ (respectively). We consider support and core intervals as confidence intervals (with a confidence level at $100 \%$ for the support and $\epsilon<<1 \%$ for the core). Simulations are performed till $t=8 \mathrm{~h}$, and the final states are used to rebuild the trapezoidal fuzzy numbers as given in Figure 11

Discussion: The example from [13] being different than the purpose treated in this paper, the capabilities of confidence based contractor are not really exploited. However, the comparison is interesting. First of all, it is important to notice that the reachability method used in [13] is more efficient than the one used here (VSPODE is dedicated to handle uncertain parameters), and thus the reachable tube seems thinner. However, the way that discretization with $\alpha$-cuts is performed in [13] leads to consider as constant a parameter during all the integration process while our approach allows all the possible values at each instant. Based on this observation, the method proposed in [13] is probably more optimistic than the confidence based propagation, as depicted in Figure 11. To conclude this discussion, our method is not dedicated to consider correlation between parameters. Furthermore, this point is not clearly treated in [13].

\subsection{Inverse problem}

The inverse problem consisting in finding the confidence level such that a constraint on reachable set can be proved is interesting. For example, a requirement in term of confidence level on the position of a robot is directly connected to the quality of the measurement devices of the robot. Therefore, if a certain sensor quality is required by the system to validate a given property (e.g. safety), it is important to be able to bound the required confidence level. 


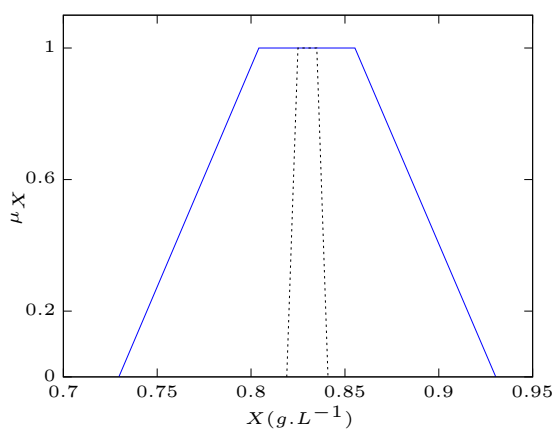

(a) Biomass concentration

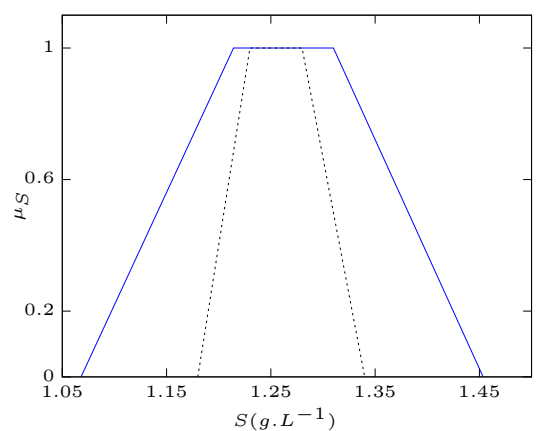

(b) Substrate concentration

Figure 11: Results for Example (11) at $t=8 \mathrm{~h}$ (in blue: the concentrations w.r.t. confidence intervals for parameters (support / core); in dashed lines: results from $[13])$.

A contractor approach associated with a forward-backward propagation (the specific contractor programming approach presented in [2] is exploited) can be used to compute the initial condition $\left[y_{0}\right]$ such that $\left[y\left(5 ;\left[y_{0}\right]\right)\right] \subset[1.5,1.6]$. After this preliminary step, the confidence coefficient is computed with: $c c=\operatorname{Pr}(x \in$ $\left.\left[y_{0}\right]\right)=\int_{\left[y_{0}\right]} f_{X}(x) d x$.

The algorithm used consists of four steps:

1. Validated simulation with $\left[y_{0}\right]$ till $t_{\text {end }}$ to obtain $\left[y\left(t_{\text {end }} ;\left[y_{0}\right]\right)\right]$

2. Intersection of $\left[y\left(t_{\text {end }} ;\left[y_{0}\right]\right)\right]$ with the goal: $\left[y\left(t_{\text {end }}\right)\right]=\left[y\left(t_{\text {end }} ;\left[y_{0}\right]\right)\right] \cap\left[y_{\text {goal }}\right]$

3. Backward propagation from $t_{\text {end }}$ to $t=0$ to obtain $\left[y\left(0 ;\left[y\left(t_{\text {end }}\right)\right]\right)\right]$

4. Computation of the confidence coefficient: $\operatorname{Pr}\left(x \in\left[y\left(0 ;\left[y\left(t_{\text {end }}\right)\right]\right)\right]\right)$

On the previous example, the backward propagation provides $\left[y_{0}\right]=$ $[0.200625,1.79937]$ which gives a confidence coefficient $c c=[0.576115,0.576464]$, i.e., a confidence level of around $57.62 \%$.

\section{Conclusion and future works}

In this paper, a novel contractor based on confidence level is proposed. It aims to reduce the pessimism of the interval approach by considering the probability density of the variables. We showed on a simple running example that our method provides results corresponding to the theory on normal density. An application to the reachability of ordinary differential equations has been proposed. The confidencebased contractor has been associated to a validated integration method to compute reachable sets for different values of confidence level. A propagation procedure allows one to propagate the contraction on initial state to the reachable set. We 
proposed to depict the different reachable sets under the form of a potential cloud. This method was tested on an example. Finally, the inverse problem consisting in computing the confidence coefficient such that a constraint on the reachable set is fulfilled has been solved.

As future work, even if no limits exist on the proposed approach in terms of problem size or considered distribution, we should apply it to more complex examples and different probability densities. Moreover, it could be interesting to exploit it in the field of robotics considering the distribution of the sensors in a control synthesis, safety verification or path planning.

\section{References}

[1] Abdallah, Fahed, Gning, Amadou, and Bonnifait, Philippe. Box particle filtering for nonlinear state estimation using interval analysis. Automatica, 44(3):807 - 815, 2008. DOI: 10.1016/j .automatica. 2007.07.024.

[2] Alexandre dit Sandretto, Julien and Chapoutot, Alexandre. Contraction, propagation and bisection on a validated simulation of ODE. In Small Workshop on Interval Methods, 2016.

[3] Alexandre dit Sandretto, Julien and Chapoutot, Alexandre. Validated explicit and implicit Runge-Kutta methods. Reliable Computing, 22:79-103, 2016.

[4] Alexandre dit Sandretto, Julien and Wan, Jian. Reachability analysis of nonlinear odes using polytopic based validated runge-kutta. In Potapov, Igor and Reynier, Pierre-Alain, editors, Reachability Problems, pages 1-14. Springer International Publishing, 2018. DOI: 10.1007/978-3-030-00250-3_1.

[5] Chabert, Gilles and Jaulin, Luc. Contractor programming. Artificial Intelligence, 173(11):1079 - 1100, 2009. DOI: 10.1016/j.artint.2009.03.002.

[6] Destercke, Sébastien, Dubois, Didier, and Chojnacki, Eric. Transforming probability intervals into other uncertainty models. In EUSFLAT 2007 proceedings, volume 2, pages 367-373, 2007.

[7] Dubois, Didier, Kerre, Etienne, Mesiar, Radko, and Prade, Henri. Fuzzy Interval Analysis. In Fundamentals of Fuzzy Sets, pages 483-581. Springer US, Boston, MA, 2000. DOI: 10.1007/978-1-4615-4429-6_11.

[8] Ferson, Scott, Kreinovich, Vladik, Grinzburg, Lev, Myers, Davis, and Sentz, Kari. Constructing probability boxes and dempster-shafer structures. Technical report, Sandia National Lab.(SNL-NM), Albuquerque, NM (United States), 2015.

[9] Fuchs, Martin. Cloud based design optimization. In IFSA/EUSFLAT Conf., pages 345-350, 2009. 
[10] Fuchs, Martin and Neumaier, Arnold. Autonomous robust design optimisation with potential clouds. Int. J. Reliability and Safety, 3:23-34, 01 2009. DOI: 10.1504/IJRS. 2009. 026833.

[11] Jaulin, Luc, Kieffer, Michel, Didrit, Olivier, and Walter, Eric. Applied Interval Analysis. Springer, 2001. DOI: 10.1007/978-1-4471-0249-6.

[12] Lohner, Rudolf J. Enclosing the solutions of ordinary initial and boundary value problems. Computer Arithmetic, page 255-286, 1987.

[13] Măceş, D Andrei and Stadtherr, Mark A. Computing fuzzy trajectories for nonlinear dynamic systems. Computers \& chemical engineering, 52:10-25, 2013. DOI: $10.1016 / \mathrm{j}$. compchemeng. 2012.11.008.

[14] Mendenhall, William, Beaver, Robert J, and Beaver, Barbara M. Introduction to Probability and Statistics. Cengage Learning, 2012.

[15] Moore, Ramon E. Interval Analysis. Series in Automatic Computation. Prentice Hall, 1966.

[16] Moore, Ramon E., Kearfott, R Baker, and Cloud, Michael J. Introduction to Interval Analysis. Siam, 2009. DOI: 10.1137/1.9780898717716.

[17] Nedialkov, N. S., Jackson, K. R., and Corliss, G. F. Validated solutions of initial value problems for ordinary differential equations. Applied Mathematics and Computation, 105(1):21-68, 1999. DOI: 10.1016/S0096-3003(98) 10083-8.

[18] Neumaier, Arnold. Clouds, fuzzy sets, and probability intervals. Reliable computing, 10(4):249-272, 2004. DOI: 10.1023/B:REOM.0000032114.08705. cd.

[19] Neyman, Jerzy. Outline of a theory of statistical estimation based on the classical theory of probability. Phil. Trans. R. Soc. Lond. A, 236(767):333380, 1937. DOI: $10.1098 /$ rsta.1937.0005.

[20] Rauh, A., Hofer, E. P., and Auer, E. Valencia-ivp: A comparison with other initial value problem solvers. In 12th GAMM - IMACS International Symposium on Scientific Computing, Computer Arithmetic and Validated Numerics (SCAN 2006), pages 36-36, 2006. DOI: 10.1109/SCAN.2006.47.

[21] Williamson, Robert C. and Downs, Tom. Probabilistic arithmetic. I. Numerical methods for calculating convolutions and dependency bounds. International Journal of Approximate Reasoning, 4(2):89 - 158, 1990. DOI: 10.1016/0888-613X (90)90022-T.

[22] Zadeh, Lotfi A. Fuzzy sets. Information and control, 8(3):338-353, 1965. DOI: $10.1016 / \mathrm{S} 0019-9958(65) 90241-\mathrm{X}$. 